\title{
Trauma Resident Exposure in Canada and Operative Numbers (TraumaRECON): a study protocol for a national multicentre study of operative, nonoperative and structured educational exposures in Canada
}

\author{
Qian Shi MD, Angela Coates MEd, Paul T. Engels MD, Timothy J. Rice MD MSc; for the Canadian \\ Collaborative on Urgent Care Surgery (CANUCS)
}

\section{Abstract}

Background: Canada's shift toward nonoperative trauma management, coupled with the implementation of competency-based medical education, has highlighted the lack of quantitative knowledge about the volume and quality of exposure to operative trauma training experiences among Canadian general surgery residents. We aim to quantify the exposure to specific operative trauma domains during residency over time and across participating Canadian training programs and to perform an environmental scan of the nonoperative clinical exposure and other formal and informal trauma education provided to general surgery residents across Canadian training programs.

Methods: Trauma Resident Exposure in Canada and Operative Numbers (TraumaRECON) is a retrospective, multicentre study of operative trauma procedures involving the participation of general surgery residents in Canada. Participating sites will populate a data abstraction form outlining operative trauma data points as abstracted from eligible trauma operative charts via each site's trauma registry. They will also complete a survey of the nonoperative clinical and other educational opportunities in trauma care to which general surgery residents are exposed in participating general surgery training programs. The primary outcome of this study will be the volume of operative trauma cases that general surgery residents are exposed to during their residency in Canada. Secondary outcomes will include the association between time of occurrence during the day for trauma operations and resident participation, operative volume stratified by postgraduate year of training, volume of missed operative trauma opportunities, volume of operative trauma cases by type, and the operative role of residents involved in trauma operations.

Interpretation: The need for competency in operative trauma management will always exist; however, with potentially limited operative trauma volume, this standard may prove difficult to achieve for the next generation of general surgery residents in Canada. Results of TraumaRECON will provide a quantitative commentary on the operative trauma volume experienced by general surgery residents in Canada to inform future teaching practices in the context of competency-based medical education.

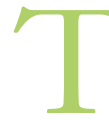
raditionally, the competency of general surgery residents in Canada was evaluated qualitatively by attending surgeons rather than by means of formal systems to quantify clinical achievements or deficiencies. Over the past 2 years, the principles of competency-based medical education have been introduced into training programs for general surgery in Canada, with the objective of using resident data in a longitudinal, levels-based approach to evaluating competencies. ${ }^{1,2}$ These changes to Canada's training approach are occurring in the context of substantial shifts in the provision of trauma care, namely the increasing use of nonoperative management. Recent original research and published commentary on trauma has posited that the wider availability of high-quality diagnostic imaging, evidence-based strategies for nonoperative management, and effective use of interventional or endovascular technology are contributing factors driving trauma care toward nonoperative management. ${ }^{3-5}$

Regardless of how trauma management evolves, there will always be a need for competent, adequately trained trauma surgeons. Given that general surgery graduates may have limited operative trauma experiences, their competency in operative trauma management is in question in Canada. ${ }^{5}$ Furthermore, competency-based medical education also challenges

Competing interests: None declared.

This article has been peer reviewed.

Correspondence to: Timothy Rice, ricet@hhsc.ca

CMAJ Open 2020. DOI:10.9778/cmajo.20190185 
the concept of competence in operative trauma management among general surgery residents, as surgical education has shifted to an educational model that assesses competency on the basis of the frequency with which residents perform essential surgical procedures, such as trauma laparotomy, and the breadth and depth of their skills. ${ }^{1}$ Without a record of, and thus insight into, the specific volume of operative trauma procedures prior residents needed to perform successfully to be considered competent by evaluators, it may prove challenging to determine a standard for operative trauma competency in the competency-based medical education model. Together, these factors challenge our ability to assess and ensure the competency of general surgery graduates in operative trauma management.

Amid the changing landscape of general surgery training, a comprehensive investigation of resident trauma experiences should be conducted. The Trauma Resident Exposure in Canada and Operative Numbers (TraumaRECON) study aims to quantify the exposure to specific operative trauma domains during residency over time and across participating Canadian training programs and to perform an environmental scan of the nonoperative clinical exposure and other formal and informal trauma education provided to general surgery residents across Canadian training programs.

\section{Methods}

This study will include both a quantitative report of resident operative trauma exposure across Canada and an environmental scan of formal trauma curricula offered by Canadian general surgery residency programs.

\section{Setting}

At present, general surgery residency programs are offered at 17 universities in 8 Canadian provinces. ${ }^{2}$ Hospital sites eligible to participate in this study must be university-affiliated Canadian level 1 trauma centres with the resources and capabilities to manage patients with multisystem trauma operatively. Sites must be affiliated with a general surgery training program accredited by the Royal College of Physicians and Surgeons of Canada and provide general surgery residents with clinical exposure to the trauma service at the site.

Eligible sites must possess an organized internal registry of patients with trauma admitted to the site within the study period or have the means to extract data about these patients from a master site registry to create a site-specific trauma registry. Sites must also be able to provide evidence of the attendance of surgeons, general surgery residents and fellows at trauma operations through medical records, as well as a record of the date, time and type of operation performed.

Twenty-two Canadian trauma centres associated with the 17 general surgery training programs currently accredited by the Royal College of Physician and Surgeons of Canada will be invited to participate in both components of this study. The list of trauma centres that will be invited to participate can be found in Appendix 1, available at www.cmajopen.ca/ content/8/4/E715/suppl/DC1.

Invitations will be sent electronically via email to the trauma medical directors for each program. The coordinating study centre, responsible for data compilation and analysis, will be located at McMaster University in Hamilton, Ontario.

\section{Design and outcomes}

The TraumaRECON Study will consist of 2 components (Figure 1): a retrospective multicentre study of all operative trauma procedures involving general surgery resident participation in Canada, and a survey of participating general surgery training programs to describe the nonoperative clinical and educational opportunities in trauma care to which general surgery residents are exposed.

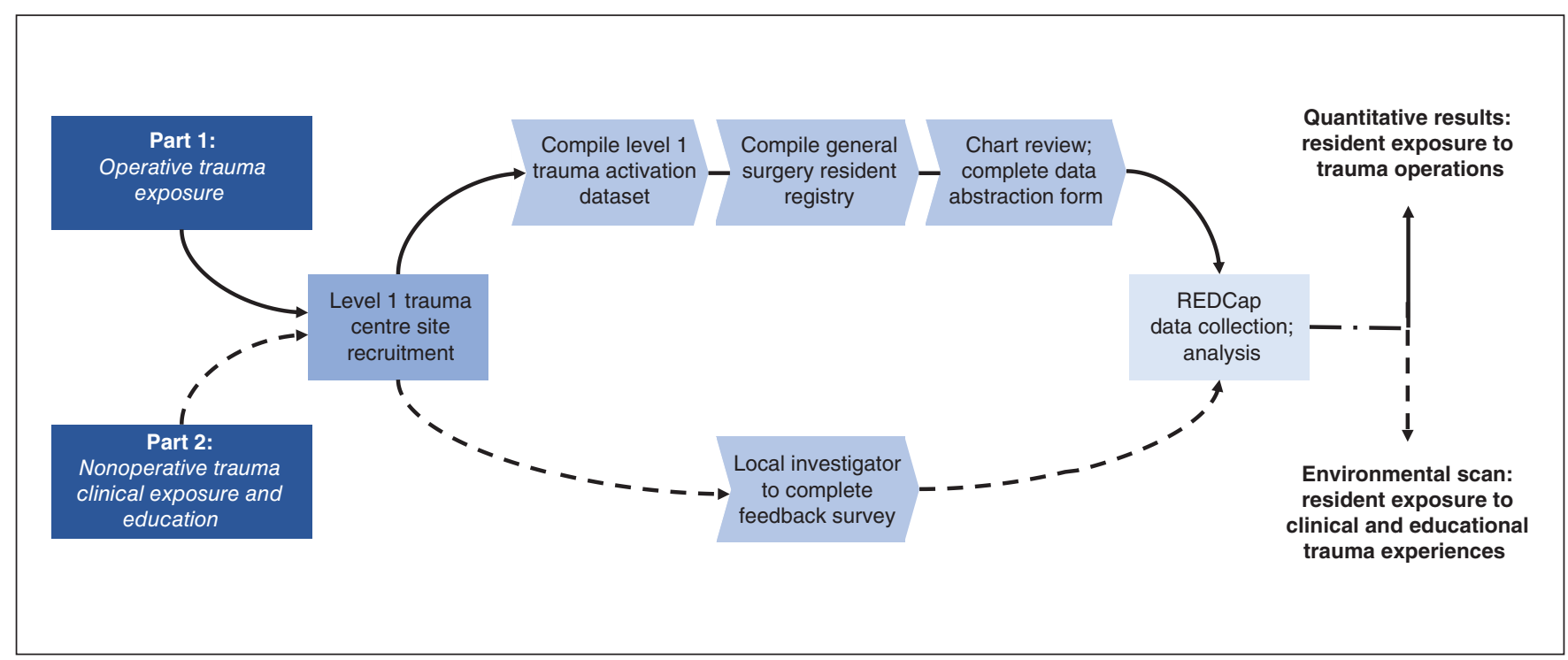

Figure 1: Overview of Trauma Resident Exposure in Canada and Operative Numbers (TraumaRECON) study. REDCap = Research Electronic Data Capture. 
In 2019, we completed a pilot study at McMaster University, which involved the first component of our proposed design. Methodology and data collection were identical to those of the protocol described in the present article. Data from the pilot study were presented at 2 peer-reviewed conferences $^{6,7}$ and a report on the study was recently published in the Canadian Medical Education Fournal. ${ }^{8}$

Our primary outcome of interest will be the volume of operative trauma cases to which general surgery residents have been exposed throughout their postgraduate training in Canada. Secondary outcomes of interest include the association between time of occurrence during the day for trauma operations and resident participation, operative participation volume stratified by postgraduate year of training, volume of operative trauma opportunities missed by residents, volume of operative trauma cases by type and the operative role of residents involved in trauma operations.

\section{Part 1: Operative trauma exposure}

At each participating site (local trauma centre), the trauma registry database will be queried to identify all patients with trauma who underwent nonorthopedic, nonneurosurgical and nonplastic operations for trauma during the study period of July 1, 2008, to June 30, 2018. This time period was chosen to capture the entire training period for a substantial number of residents (5-yr training period occurring within study period) and to allow us to assess temporal trends. These patients will form the study cohort.

At each site, data will be abstracted at the level of each patient chart to obtain details about the operations performed and the presence of staff and resident surgeons. A comprehensive listing of general surgery residents in the local residency program during the study period will be obtained from the local residency program office. The resident surgeons identified in the trauma operations will be cross-referenced with their postgraduate year of training according to the information provided by the residency program office. Each site will therefore be able to create a local cohort of all patients who were treated operatively for trauma, with corresponding data identifying the postgraduate year of training of each general surgery resident present at the operation.

Because patients with trauma may undergo multiple eligible surgeries, the unit of analysis for the study is the surgical event. All general surgery, thoracic, cardiac, head-and-neck, bladderrelated urologic and abdominal vascular operative procedures for trauma, as well as thoracotomies taking place in the emergency department, will be eligible for this study. All singleservice procedures for trauma will be excluded (e.g., orthopedics, plastic surgery, obstetrics and gynecology, otolaryngology, ophthalmology and neurosurgery). All procedures done at the bedside in the emergency department or intensive care unit (e.g., tube thoracostomy, tracheostomy and percutaneous endoscopic gastrostomy tube insertion) will also be excluded.

For each eligible surgery, data on patient demographic characteristics, mechanism, time of operation, index or repeat operation, and the specific operation performed will be obtained from the trauma registry and chart review. The types of procedures will be cross-referenced to those listed in Surgical Procedures List A and Surgical Procedures List B of the Royal College of Physicians and Surgeons of Canada's Objectives of Training in the Specialty of General Surgery document. ${ }^{9}$

\section{Part 2: Nonoperative trauma clinical exposure and other education}

Each participating site will complete a survey asking for details regarding the typical general surgery resident's clinical exposure to patients with trauma during their residency. The survey will include questions about the number of dedicated trauma service rotations, the number of rotations spent at a hospital that takes care of patients with trauma, on-call responsibilities caring for these patients, and the involvement in trauma resuscitation and inpatient care. In addition, the survey will ask about other formal and informal educational opportunities provided to residents, such as courses from the American College of Surgeons (e.g., Advanced Trauma Life Support, Advanced Trauma Operative Management, Advanced Surgical Skills for Exposure in Trauma) and the Definitive Surgical Trauma Care Course, ${ }^{10}$ academic half-day topics, the presence and frequency of trauma rounds and other similar such exposures.

The survey was developed in an iterative fashion on the basis of expert consensus by the members of the Canadian Collaborative on Urgent Care Surgery (CANUCS; https:// canucs.ca/), a research collaborative born out of the Acute Care Surgery Committee of the Canadian Association of General Surgeons. It was pilot tested and refined by representatives from multiple provinces. The full survey can be found in Appendix 2, available at www.cmajopen.ca/content/8/4/ E715/suppl/DC1.

\section{Sample size}

According to available data from the Canadian Resident Matching Service match (2008-2018), the average number of filled general surgery residency positions is 93 annually. ${ }^{11}$ By auditing all training sites affiliated with the Royal College of Physicians and Surgeons of Canada over the study period, we anticipate having a maximum of 930 resident-years for analysis, which should include the complete training duration of 6 cohorts of residents over the study period, or approximately 558 residents (Figure 2).

\section{Data collection}

\section{Part 1: Operative trauma exposure}

The names of residents from each participating Canadian general surgery program who were enrolled in the residency program during the study period will be obtained from the general surgery residency program office at each site.

Participating trauma centres will receive a data abstraction form listing data elements for abstraction (Table 1). This form was used successfully during the pilot study. ${ }^{8} \mathrm{~A}$ compilation of trauma operations will be extracted from each trauma centre's patient database, and chart reviews 


\begin{tabular}{|c|l|l|l|l|l|l|l|l|l|l|}
\hline $\begin{array}{l}\text { Resident } \\
\text { cohort }\end{array}$ & 200809 & $2009 / 10$ & $2010 / 11$ & $2011 / 12$ & $2012 / 13$ & $2013 / 14$ & $2014 / 15$ & $2015 / 16$ & $2016 / 17$ & $2017 / 18$ \\
\hline 1 & $\bullet$ & $\bullet$ & $\bullet$ & $\bullet$ & $\bullet$ & & & & & \\
\hline 2 & & $\bullet$ & $\bullet$ & $\bullet$ & $\bullet$ & $\bullet$ & & & & \\
\hline 3 & & & $\bullet$ & $\bullet$ & $\bullet$ & $\bullet$ & $\bullet$ & & & \\
\hline 4 & & & & $\bullet$ & $\bullet$ & $\bullet$ & $\bullet$ & $\bullet$ & & \\
\hline 5 & & & & & $\bullet$ & $\bullet$ & $\bullet$ & $\bullet$ & $\bullet$ & \\
\hline 6 & & & & & & $\bullet$ & $\bullet$ & $\bullet$ & $\bullet$ & $\bullet$ \\
\hline
\end{tabular}

Figure 2: Theoretical distribution of resident cohorts that completed training during the study period.

will be conducted to exclude operative traumas ineligible for the study.

Depending on the centre, chart reviews will be conducted by residents, attending surgeons or research assistants. Eligible operative traumas will be included on the data abstraction form. Residents identified on the data abstraction form will be cross-referenced with their program database to determine their year of training at the time of the operation. Resident, patient and surgeon identification will be anonymized locally by each participating site.

This information will be collected on a standardized spreadsheet and will be sent to the coordinating centre in accordance with the requirements of the local research ethics board and data-sharing agreement. Single-site data will be combined into a master cohort for analysis at the coordinating site (McMaster University). Data will be deidentified locally before transmission to the coordinating site via secured data transfer processes.

\section{Part 2: Nonoperative trauma clinical exposure and other education}

Surveys will be sent to the local investigators at the participating sites. Local investigators will be asked to complete the survey with input as required from the medical director for each trauma centre and the general surgery residency program director. Local investigators will include regional trauma medical directors and trauma surgeon members of CANUCS. The local investigator will be asked to take responsibility for the accuracy of the data supplied.

Survey responses from all sites will be anonymized at the local sites, then gathered and compiled into a master response list at the coordinating centre at McMaster University.

\section{Statistical analysis}

\section{Part 1: Operative trauma exposure}

Trauma operations will be categorized by weekdays or weekends and by start time (i.e., 0700 to 1659 as daytime; 1700 to
2259 as evening; and 2300 to 659 as overnight). The operative role of the resident will be inferred using an algorithm developed by the study team. The algorithm includes factors such as the number of staff surgeons present, the training level of the resident and the presence of additional residents or fellows (Appendix 3, available at www.cmajopen.ca/ content/8/4/E715/suppl/DC1). We will also quantify "potential missed trauma exposure opportunity," defined as any operative trauma that did not involve at least 1 general surgery resident.

Descriptive statistics will be presented as frequencies for categorical variables and means with standard deviation or medians with quartile ranges for continuous variables (e.g., mean exposure of residents to trauma operations by study site, and according to type of operation, day and time of operation and operative role). The mean and median number of trauma operations in which residents participated will be reported by postgraduate year of training.

The primary outcome of interest in this study will be dichotomous: fewer than 10 operative trauma cases versus 10 or more operative trauma cases during a resident's entire 5 -year general surgery residency training period. The threshold of 10 cases was selected because this represents the existing standard for adequacy based on the Accreditation Council for Graduate Medical Education case minimums in the United States. $^{12}$

A hierarchical multivariable logistic regression analysis will be performed to determine factors associated with an operative trauma volume of 10 or more cases. All variables associated with resident operative trauma volume entered into the model will be determined a priori. Model fit will be assessed using the Akaike information criterion and the HosmerLemeshow goodness-of-fit test. Multicollinearity will be assessed using the variance inflation factor. Deviance residuals will be assessed for the presence of influential outliers.

Data analysis was projected to start and be completed between 2020 and 2021, but because of the widespread suspension of all research activities during the global 
Table 1 (part 1 of 2): Summary of data points to be abstracted for Part 1 of the TraumaRECON study, with sample data for a patient who had 2 visits to the operating room during his hospital admission

\begin{tabular}{|c|c|c|}
\hline Data point & $\begin{array}{l}\text { Sample data for } \\
\text { operation } 1^{*}\end{array}$ & $\begin{array}{l}\text { Sample data for } \\
\text { operation } 2^{*}\end{array}$ \\
\hline Participating site ID & 7 & 7 \\
\hline Patient ID & 25 & 25 \\
\hline $\begin{array}{l}\text { Date of arrival at lead trauma centre } \\
\text { (must be between July } 1,2008 \text {, and June } 30,2018 \text { ) }\end{array}$ & Mar. 25, 2015 & Mar. 25, 2015 \\
\hline Date of discharge or death from lead trauma centre & Apr. 15, 2015 & Apr. 15, 2015 \\
\hline Patient age, yr & 28 & 28 \\
\hline Patient sex & M & M \\
\hline Injury type (blunt, penetrating, other) & Blunt & Blunt \\
\hline Injury Severity Score & 16 & 16 \\
\hline Date of OR visit (must be between July 1,2008 , and June 30,2018 ) & Mar. 25, 2015 & Mar. 27, 2015 \\
\hline Start time of operation & 1423 & 830 \\
\hline \multicolumn{3}{|l|}{ Laparotomy or laparoscopy } \\
\hline Index laparotomy & 1 & 0 \\
\hline Second-look laparotomy & 0 & 1 \\
\hline Index laparoscopy & 0 & 0 \\
\hline Second-look laparoscopy & 0 & 0 \\
\hline Laparoscopy converted to laparotomy & 0 & 0 \\
\hline \multicolumn{3}{|l|}{ Abdominal intraoperative procedures } \\
\hline Splenectomy & 0 & 1 \\
\hline Liver resection or repair & 0 & 0 \\
\hline Bowel resection or repair & 0 & 0 \\
\hline Diaphragm repair & 0 & 1 \\
\hline $\begin{array}{l}\text { Retroperitoneal exploration (defined as use of left- or right-sided } \\
\text { medial visceral rotation) }\end{array}$ & 0 & 0 \\
\hline Pancreas resection & 0 & 0 \\
\hline Duodenal repair & 0 & 0 \\
\hline Renal resection or repair & 0 & 0 \\
\hline Bladder repair & 0 & 0 \\
\hline Major abdominal vascular repair (defined as repair of named vessel) & 0 & 0 \\
\hline \multicolumn{3}{|l|}{ Any type of thoracic operation } \\
\hline Thoracotomy performed in ED & 0 & 0 \\
\hline \multicolumn{3}{|c|}{ Date of thoracotomy performed in ED (between July 1,2008 , and June 30,2018 ) } \\
\hline \multicolumn{3}{|c|}{$\begin{array}{l}\text { Time of thoracotomy performed in ED (if procedure start time not recorded, use } \\
\text { patient's time of arrival in ED) }\end{array}$} \\
\hline Sternotomy performed in OR & 0 & 0 \\
\hline Thoracotomy performed in OR & 0 & 0 \\
\hline VATS $^{*}$ & 0 & 0 \\
\hline VATS converted to thoracotomy & 0 & 0 \\
\hline \multicolumn{3}{|l|}{ Thoracic intraoperative operation } \\
\hline Repair of heart & 0 & 0 \\
\hline Lung resection or repair & 0 & 0 \\
\hline Major thoracic vascular repair & 0 & 0 \\
\hline Any type of neck exploration & 0 & 0 \\
\hline
\end{tabular}




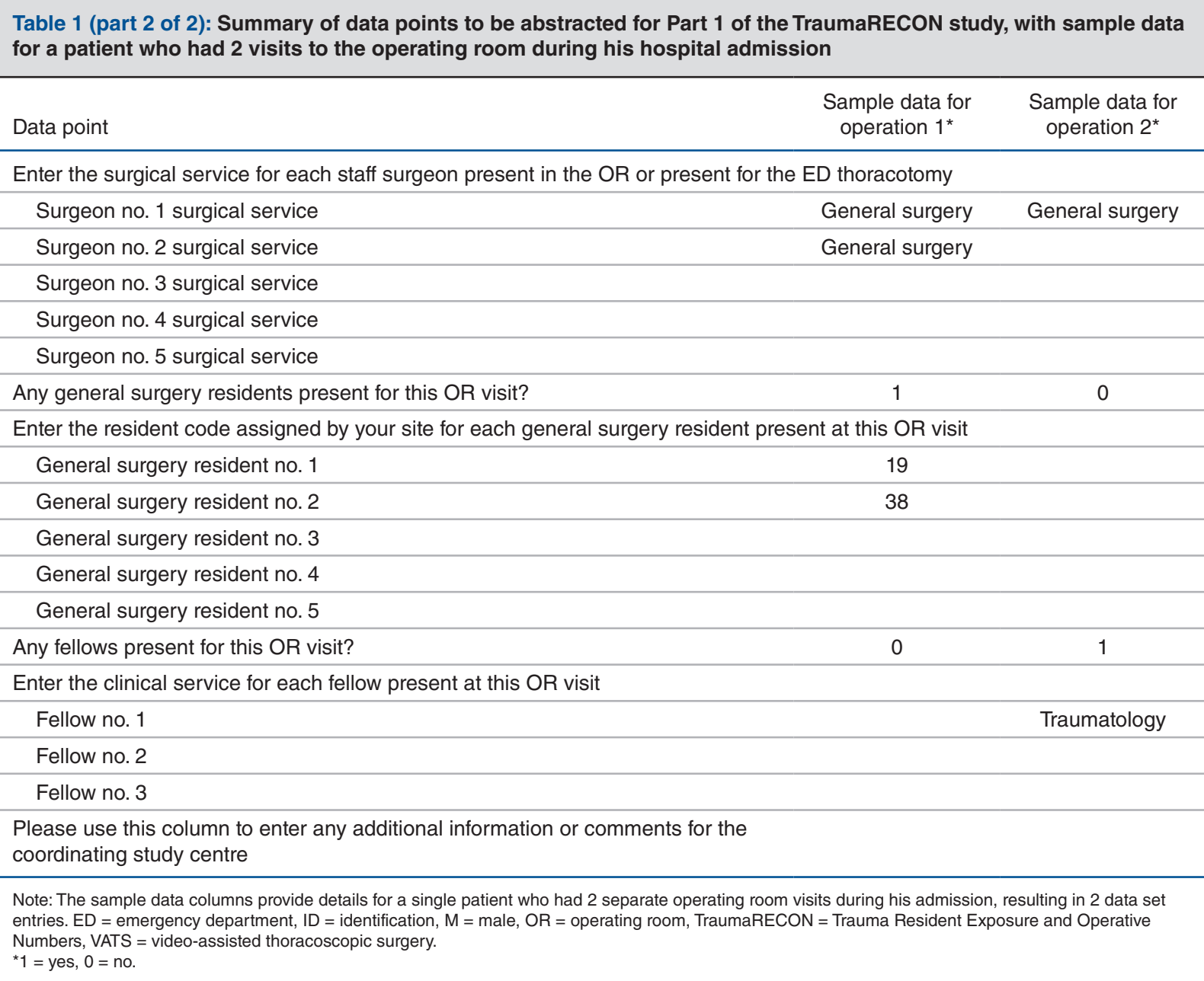

COVID-19 pandemic, this timeline will be pushed back, depending on when each institution allows research activities to resume.

\section{Part 2: Nonoperative trauma clinical exposure and other education}

Resident program data will be described according to the formal and informal trauma curriculum factors included in the survey. Programs will be anonymized and information will be presented in such a way that no program can be identified.

Data will be analyzed using SPSS version 25 (IBM Corporation).

\section{Ethics approval}

The Hamilton Integrated Research Ethics Board provided approval for the completed pilot study performed at McMaster University. Approval has been obtained from this board for this multicentre study. Approval will be required from the local research ethics board at each participating site; participating centres will be responsible for the application to their research ethics board and will use this study protocol and data abstraction forms as references.

\section{Interpretation}

Results from this study will be used to determine a baseline volume of operative trauma procedures performed by general surgery residency graduates in Canada. This information will inform the competency-based medical education process of identifying potential target volumes for operative trauma procedures. It will also be used to determine the operative trauma volumes across Canadian level 1 trauma centres to assess their existing capacity to provide training in trauma care. In addition, the results from this study will be used to identify opportunities to enhance trauma education resources in general surgery residency programs in Canada.

The changing landscape of both trauma management and competency-based medical education in general surgery residencies necessitates an investigation into the degree of trauma operative exposure, clinical experience and formal teaching that general surgery residents receive at training programs accredited by the Royal College of Physicians and Surgeons of Canada. The TraumaRECON study aims to provide insight into the specific trauma experiences, procedures and roles general surgery residents are 
offered throughout their training in Canada. Results of the TraumaRECON study will be shared with all Canadian general surgery residency programs as well as with relevant trauma and educational organizations, including the Royal College of Physicians and Surgeons of Canada and the Trauma Association of Canada. Results will be disseminated via conference presentations and email notices to program directors and trauma medical directors. We hope that the dissemination of our results will engage leaders in Canadian trauma education to refine standards for operative trauma education in the context of changing operative volumes and competency-based medical education.

\section{Limitations}

Our study is vulnerable to the biases associated with a retrospective design. Each participating site will be asked to include all consecutive surgical events in the trauma registry for the 10-year period to minimize risk of selection bias.

Retrospective cohort studies are prone to information or misclassification bias. We suggest that this is minimized through the use of trauma registry data that is abstracted by coding experts and is subject to ongoing reliability testing. For additional chart-abstracted fields, sites will perform a 5\% reabstraction on a random sample of every 100 abstracted charts to assess interobserver agreement and ensure the accuracy and quality of chart abstraction data. In addition, data abstractors at all participating sites will be blinded to the study hypothesis to minimize information bias.

Our survey may be vulnerable to social desirability bias. Given that questionnaire respondents are all faculty - representing their respective academic institutions - there may be reluctance to acknowledge any inherent weaknesses in their program, biasing responses toward more favourable responses. To minimize bias, respondents will be informed that all responses will be deidentified and kept strictly confidential with the exception that the principal investigators will have access to site-specific data.

\section{Conclusion}

The results of this study will provide a quantitative and qualitative understanding of the current status of operative trauma training for general surgery residents in Canada. We believe our results will inform future trauma teaching practices in the context of competency-based medical education and contribute to the training of general surgery graduates competent in operative trauma care.

\section{References}

1. Competency by design: Canada's model for competency-based medical education. Ottawa: The Royal College of Physicians and Surgeons of Canada. Available: www. royalcollege.ca/rcsite/cbd/competence-by-design-cbd-e (accessed 2019 Oct. 16).

2. General surgery: program directors. Ottawa: The Royal College of Physicians and Surgeons of Canada. Available: www.royalcollege.ca/rcsite/documents/ arps/gen-surgery-e (accessed 2020 Aug. 1).

3. Strumwasser A, Grabo D, Inaba $\mathrm{K}$, et al. Is your graduating general surgery resident qualified to take trauma call? A 15-year appraisal of the changes in general surgery education for trauma. 7 Trauma Acute Care Surg 2017;82:470-80.

4. Musonza T, Todd SR, Scott B, et al. Trends in resident operative trauma: How to train future trauma surgeons? Am 7 Surg 2019;218:1156-61.

5. Engels PT, Bradley NL, Ball CG. The current state of resident trauma training: Are we losing a generation? Can 7 Surg 2018;61:153-4.

6. Versolatto A, Shi Q, Coates A, et al. Trauma surgery exposure among general surgery residents at McMaster University: a retrospective case log analysis. Can 7 Surg 2019;62(Suppl 2):S20.

7. Shi Q, Versolatto A, Coates A, et al. Trauma surgery exposure among general surgery residents at McMaster University: a retrospective case log analysis. International Conference on Residency Education; 2019 Sept. 26-28, 2019; Ottawa. Ottawa: The Royal College of Physicians and Surgeons of Canada. Available: www. royalcollege.ca/rcsite/documents/icre/2019-icre-jgme-abstract-book-jgme .pdf (accessed 2020 Mar. 4).

8. Engels P, Versolatto A, Shi Q, et al. Cause for concern: resident experience in operative trauma during general surgery residency at a Canadian centre. CMEF 2020 Aug. 4. doi: $10.36834 / \mathrm{cmej} .69323$.

9. Objectives of training in the specialty of general surgery. Ottawa: The Royal College of Physicians and Surgeons of Canada; 2017. Available: www.royalcollege.ca/ rcsite/documents/ibd/general-surgery-otr-e (accessed 2019 Oct. 16).

10. Definitive Surgical Trauma Care (DSTC ${ }^{\mathrm{TM}}$ ) courses. Zurich (Switzerland): International Association for Trauma Surgery and Intensive Care. Available: https://iatsic.org/DSTC/ (accessed 2020 Aug. 1)

11. R-1 data and reports. Ottawa: Canadian Resident Matching Service. Available: www.carms.ca/data-reports/r1-data-reports/ (accessed 2020 Aug. 1).

12. Defined category minimum numbers for general surgery residents and credit role: Review Committee for Surgery. Chicago: Accreditation Council for Graduate Medical Education; 2019. Available: www.acgme.org/Portals/0/DefinedCategory MinimumNumbersforGeneralSurgeryResidentsandCreditRole.pdf (accessed 2020 Aug. 1).

Affiliations: Department of Surgery (Shi, Coates, Engels, Rice), McMaster University; Trauma Program (Coates, Engels, Rice), Hamilton General Hospital, Hamilton Health Sciences, Hamilton, Ont.

Contributors: All authors contributed to conceptualization, methodology, data curation, formal analysis, investigation, validation, visualization, writing the article and revising it critically for important intellectual content. All authors approved the final version of the article to be published and agreed to be accountable for all aspects of the work.

Funding: The study received funding from an education research grant from McMaster Surgical Associates.

Data sharing: The data collected by this study will be subject to research ethics board policies and data-sharing agreement contracts and hence not available to those outside of the TraumaRECON study team.

Acknowledgements: The TraumaRECON study is supported formally by the Canadian Collaborative on Urgent Care Surgery (CANUCS) of the Canadian Association of General Surgeons.

Supplemental information: For reviewer comments and the original submission of this manuscript, please see www.cmajopen.ca/content/8/4/ E715/suppl/DC1. 